\title{
Medical Device Development: The Challenge for Ergonomics
}

\section{Jennifer L Martin ${ }^{1}$, Beverley J Norris ${ }^{2}$, Elizabeth Murphy ${ }^{3}$, John A}

Crowe $^{1}$

${ }^{1}$ School of Electrical and Electronic Engineering, The University of Nottingham, University Park, Nottingham, NG7 2RD. UK

${ }^{2}$ National Patient Safety Agency, 4-8 Maple Street London, W1T 5HD. UK

${ }^{3}$ School of Sociology and Social Policy, The University of Nottingham, University Park, Nottingham, NG7 2RD. UK

\section{The published version of this paper can be found at:}

Martin, J.L., Norris B.J., Murphy E., Crowe, J.A. (2008) Medical Device Development: The Challenge for Ergonomics, Applied Ergonomics 39 (3), 271-283

http://www.elsevier.com/wps/find/homepage.cws_home 
Abstract: High quality, well designed medical devices are necessary to provide safe and effective clinical care for patients as well as to ensure the health and safety of professional and lay device users. Capturing the user requirements of users and incorporating these into design is an essential component of this. The field of ergonomics has an opportunity to assist, not only with this area, but also to encourage a more general consideration of the user during medical device development. A review of the literature on methods for assessing user requirements in engineering and ergonomics found that little published work exists on the ergonomics aspects of medical device development. In particular there is little advice available to developers on which issues to consider during design and development or recommendations for good practice in terms of the methods and approaches needed to capture the full range of user requirements. The Multidisciplinary Assessment of Technology Centre for Healthcare (MATCH) is a research collaboration that is working in conjunction with industrial collaborators to apply ergonomics methods to real case study projects with the ultimate aim of producing an industry-focused guide to applying ergonomics principles in medical device development.

Keywords: Medical Device, Patient Safety, User Requirements

\section{Introduction to the role of ergonomics in medical device design}

To provide safe and high quality care for patients the healthcare industry requires clinically effective and well designed medical devices. The design of devices should take account of the environment in which they are required to function and should support the working patterns of professional users and the lifestyles of patients and carers. Medical devices are a diverse group of products that ranges from simple items such as sticking plasters to complex devices such as heart by-pass machines (table 1). 
The last decade has seen an increased focus on the design of medical devices, specifically in relation to patient safety, and a number of initiatives have been set up with the aim of improving such aspects. In 2001 The National Patient Safety Agency (NPSA) was set up to improve the safety and quality of care in the UK National Health Service through the reporting, analysing and learning from adverse incidents and 'near misses'. In addition, the Design for Patient Safety initiative, which was funded by the UK Department of Health and the Design Council, provided design recommendations for equipment and packaging to reduce medical errors in the healthcare industry (Buckle et al, 2003). Ergonomics/human factors has played a significant role in both of these initiatives and a recent paper in this journal highlighted the contribution that the discipline can make towards improved safety in healthcare, particularly with regard to the design of medication packaging (Buckle et al, 2006).

The aims of this paper are two-fold. First, it presents an analysis of the methods that have been developed for assessing user needs in medical devices which draws upon a recently completed review of the literature on methods for assessing user requirements in engineering and ergonomics. Secondly, it examines the factors that make capturing user requirements during medical device development unique and challenging in what is a relatively new domain for ergonomics.

The recognition of the role of good design in improved patient safety has resulted in a number of studies investigating the usability of individual medical devices, most notably infusion pumps (Garmer et al., 2002a; Liljegren et al., 2000; Lin et al., 1998; Obradovich and Woods, 1996). In general, however, ergonomics principles and approaches are currently under-used in medical device development, especially considering the current focus on the importance of the user in other design domains such as consumer products and occupational environments.

Producing a medical device that can truly be deemed to meet the requirements of the user requires attention to many different factors. Although addressing clinical needs, human error and patient safety are critically important, satisfying user requirements during medical device 
development goes far beyond this. Devices must be designed to account for the working patterns of the users if they are to be used efficiently and effectively; users should not have to modify the device to enable it to be utilised and the design should encourage continued use if treatment or intervention protocols are to be successful. The system within which the device will be used must be considered. The healthcare sector is extremely complex and characterised by multiple users; varied healthcare settings from emergency to acute to home; remote purchasing and patient variability where factors such as type of clinical condition, health status and disability are added to the usual multiplicity of individual characteristics.

Developers, including those with a clinical background, may struggle to appreciate that user requirements encompass more than just clinical effectiveness and that for any one device there will be a number of different users to consider, including doctors, nurses, technicians, maintenance staff, patients and carers. In the majority of cases, the users of any one device will include the patient (the recipient of the care provided by the device) as well as those who operate it, which may include a number of different people, both clinical and non-clinical, and intended, trained operators plus others delivering care who may not be intended operators. The fact that the needs of multiple types of users must be considered and collected during design is one that differentiates medical devices from many other ergonomics domains. Furthermore, once the relevant information has been collected this must then be organised into a specification for the design of the device: a topic that is covered in detail by Shefelbine et al. (2002).

The picture is further complicated by the reimbursement procedures within the healthcare sector where purchasers are rarely the primary users of the device. Procurement decisions are often made remotely from the point of use, for example centrally within hospitals or trusts, regionally within procurement centres, or even nationally. Remote purchasing means that the factors such as usability and satisfaction may not be acknowledged. Those in charge of procurement may not be aware of the realities of usability and safety associated with device design and it can be difficult to establish channels for this information to be accessed. Making a case for usability 
when it implies added cost will be further hindered by this distance between those using the device and procurement. In the UK, Collaborative Procurement Hubs were recently set up with the aim of making more intelligent decisions on purchasing. As yet, these hubs are concerned solely with cost: they are currently focused on minimising costs and breaking down barriers associated with silo budgeting. In the future, however, there may be scope for these organisations to encourage device developers to adopt ergonomic principles more thoroughly by considering usability and other user issues when making purchasing decisions.

\section{Medical Device Regulation}

To ensure the safety of patients, clinical users and associated third parties, medical devices are subject to complex regulations. Conformity assessment is conducted before and after a device is placed on the market, and devices are also subject to post-market surveillance once they are in use. It is the responsibility of the medical device manufacturer to ensure device conformity.

Medical devices are a diverse group of products and are divided into specific Classes of device, for which the regulatory requirements will differ. Devices are classified by the different regulatory bodies according: to the potential hazards of using the device or the device failing; the duration of contact with the body; degree of invasiveness; and local versus systematic effects. Although classification systems are not universally agreed across the world, in general devices are categorised according to the level of risk; the higher the risks associated with a device, the higher the device class and the greater the level of assessment required by regulatory bodies.

Currently, medical device regulations vary considerably across the world, making compliance a complex and difficult process. Two of the most important regulations for developers to consider are the EC Medical Device Directive 93/42/EEC and the US Food and Drug Administration regulations, as compliance with these are required to market and sell devices in the European Community (via the CE mark) and the USA respectively. The main focus of these, and other 
medical device regulations across the world, is on risk management, the aim being to ensure that devices do not compromise either the clinical condition or the safety of patients or the health and safety of users. Developers must eliminate, or reduce as far as possible, the risks associated with their device and where appropriate take adequate protection measures: "the devices must be designed and manufactured in such a way that, when used under the conditions and for the purposes intended, they will not compromise the clinical condition or the safety of patients, or the safety and health of users" (Medical Device Directive 93/42/EEC). Aspects of design that may pose particular threats to users such as electrical safety and radiation are highlighted.

The MDD 93/42/EEC also stipulates that "Devices must be designed and manufactured in such a way as to remove or minimise as far as is possible the risk of injury, in connection with their physical features, including the volume/pressure ratio, dimensional and where appropriate ergonomic features", a rather non-specific statement in which usability is notably absent. The US FDA is more stringent and requires developers to demonstrate that human factors principles have been applied during design to identify, understand and address use-related hazards and manufacturers have to provide evidence of this during the pre-market review process. The FDA also provides some guidance on how to adopt ergonomics principles into the design process (Sawyer, 1996).

In 1993 the Global Harmonization Task Force (www.ghtf.org) was established, this is a voluntary group of representatives from national medical device regulatory authorities and the medical device industry, the five founding members are the European Union, Japan, Australia, Canada and the USA. The GHTF aims to encourage convergence in regulatory practices and facilitate international trade by publishing and disseminating harmonised guidance on regulatory practices for medical devices. The GHTF also recommends an increased use of international standards in regulation as "International standards are a building block for harmonized regulatory processes to assure the safety, quality and performance of medical devices" (Role of Standards in the Assessment of Medical Devices GHTF-SG1-N012R10, 2000). The intention is 
that this will result in a system where regulators from different countries recognise adherence to standards as meeting the necessary regulations, and standards bodies will consult with regulators when developing or revising standards relating to medical devices.

One standard that many medical device developers are likely to encounter is IEC 60601-1: General Requirements for Safety of Electrical Medical Equipment. This standard is identical to the equivalent European standard (EN) and the British standard (BS). Usability and other usercentred issues are covered in the collateral standard: 60601-1-6, which specifies the usability requirements for safety of electrical medical devices. This collateral standard requires developers to adopt a usability engineering process to ensure medical electrical equipment safety and provides brief guidance on how to implement and execute such a process, "The manufacturer should conduct iterative design and development. Usability engineering should begin early and continue through the equipment design and development lifecycle" (IEC 606011-6). Developers must keep records of their design processes to demonstrate that usability has been considered throughout. This should include the definition of a usability specification and usability goals at the beginning of development which can then be evaluated during the usability verification process. A number of ergonomics methods and techniques are suggested to evaluate and demonstrate device usability many of which will be covered later in this paper. A new standard is currently in development which, when published, will replace IEC 60601-1-6. IEC 62366 Medical Devices - General Requirements for Safety - Usability will extend the requirement for manufacturers to consider usability to all medical devices rather than just electrical medical equipment as currently is the case.

Other medical device standards that have ergonomics requirements are:

- AAMI HE74:2001: Human Factors Design Process for Medical Devices

- IEC 60601-1-8:2003 (Medical electrical equipment - Part 1-8: General requirements for safety - Collateral standard: General requirements, tests and guidance for alarm systems in medical electrical equipment and medical electrical systems) 
- ISO 14971:2000, Medical devices - Application of risk management to medical devices (2003)

- BS 7000-6:2005 Design management systems. Managing inclusive design.

The increase in focus on good design for improved usability and patient safety and the resultant increase in regulatory requirements is another pressure on medical device developers in an industry that is already extremely complex. The regulations and standards rightly concentrate on the risks to safety associated with a device, however, as discussed earlier, user requirements encompass much more than this, and focusing solely on compliance may mean that other important aspects are overlooked. For example, it is critical to involve and consider users at the concept and pre-concept stage to ensure that the device produced is the right device, that is, a device that will meet an un-met (or poorly-met) need. Producing a device that is clinically effective and safe is not sufficient. A well conceived and well designed device will help healthcare professionals carry out their work effectively and efficiently and enable patients to have improved health outcomes; a better quality of life; to become (or remain) more independent and to retain their dignity: this is a device that can truly be deemed to meet the requirements of its users. The field of ergonomics has an opportunity to assist with these issues and encourage a more general consideration of the user during medical device development.

The Multidisciplinary Assessment of Technology Centre for Healthcare (MATCH) is an Innovative Manufacturing Research Centre (IMRC) funded by the Engineering and Physical Sciences Research Council (EPSRC) and The Department of Trade and Industry (DTI). A collaboration between five UK universities, MATCH aims to support the healthcare sector by creating methods to assess the value of medical devices from concept through to mature product. Although the MATCH research is being performed within an academic framework, the emphasis is on working with research partners such as the National Patient Safety Agency (NPSA) and industrial collaborators to solve real problems. 


\section{Review of ergonomics methods in medical device design}

A structured literature review was performed to identify, summarise and appraise the published evidence on the methods and tools used for the capture of user requirements and to determine their potential for use within medical device development. The aims of the review were to:

- Map the extent of ergonomics research in the domain of medical devices and healthcare

- Assess the usability of the reported methods, either by professional ergonomists or by the medical device industry

- Identify the type, and extent of, adaptations and considerations necessary to make standard methods applicable to medical device development

- Identify the successes and barriers to assessing user requirements in medical device development as reported in the literature

- Use the results to identify a route to increasing the consideration of user requirements in medical device design

10 online bibliographic databases were used to obtain the literature (table 2). As no validated search strategies were available for the databases and they did not use an indexing method, an iterative and flexible search strategy was used. A list of keywords was compiled and this was added to throughout the review as more methods and tools were identified. Each database required different searching methods and, therefore, the keywords had to be applied differently in each case Boolean operators, quotation marks, wildcards and truncations symbols were used when appropriate.

Where available, examples of how methods have been applied to the development and evaluation of medical devices are discussed. The appropriateness of different methods for particular stages of the design cycle and their relative costs in terms of both time and money, which in business are the same thing, are also examined. Given the very real problems that are unique to the healthcare industry: namely limited access to the contexts of use as well as to the 
end users themselves - especially in acute or emergency settings - the use of proxies is also discussed.

A total of 120 papers were reviewed which covered general product development as well as medical devices development. The literature was primarily concerned with the application of user-centred design and the methods used within it. A number of published articles were identified that describe the application of user-centred design for medical devices, with the specific aim of improving usability in order to reduce medical error (Cohen, 1993; Leape, 1994; Lin et al., 1998; Obradovich and Woods, 1996; Salvemini, 1999). This illustrates the current focus in this area.

Ergonomic issues and user requirements appear to be considered and captured mainly during the design and evaluation stage of the product lifecycle. Little published work was identified where developers had included the user in other stages of the product cycle such as the concept stage. Indeed, currently, users are generally not brought into the developmental process until after the design brief for a new product has been produced. This may be because medical devices are frequently technology driven rather than resulting from an identified un-met need.

The literature review identified more than thirty methods that have been used to capture user requirements. However, many of these methods were just slight variations of other more established methods and the majority of papers identified utilised the commonly used, well established methods. Methods that were used in at least five papers are now presented, along with a discussion of the type of data they generate, specific considerations for their use in medical device development and their potential value for improved usability and user satisfaction.

\subsection{Contextual Inquiry}


Contextual Inquiry $(\mathrm{Cl})$ is an adaptation of "ethnographic research methods to fit the time and resource constraints of engineering" (Holtzblatt and Beyer, 1993 p.93) and may be an attractive option for product developers due to its pragmatic nature. With its emphasis on discovery, contextual inquiry (also known as contextual observation) is particularly suited to gathering information to inform the design of a new product or the re-design of an existing product. $\mathrm{Cl}$ is a cheaper and quicker alternative to traditional ethnography; Holtzblatt and Beyer argue that ethnography can fail to provide meaningful input into design as it can be difficult to translate the results into well defined user requirements due to its open-ended nature. Contextual inquiry is more focused, the observer/interviewer shadowing the worker as they complete their tasks eliciting information by asking the worker questions about what is happening; why it is happening and their opinions on how tasks could be improved. Holtzblatt and Beyer state that "people are adaptable and resourceful creatures - they invent many workarounds and quick fixes to problems and then forget they invented the workaround. Even the details of everyday work become second nature and invisible" (Holtzblatt and Beyer 1993 p93). Contextual inquiry ensures that the designer and the user work together to discover important information that may be implicit in the users' mind but may otherwise remain undiscovered.

We identified two studies that used $\mathrm{Cl}$ for a medical application. Coble et al. (1997) used this method as part of a participatory design approach to the development of a new clinical workstation software program. The aim of this program was to provide clinical staff with access to centrally held patient information such as radiology, pathology or laboratory test results, when treating patients either in the hospital, the office or the community. The target users for this device were physicians, especially those working in the community. The key challenge of this project was to "understand the users and their tasks and to represent this understanding in a way that supports effective system development” (Coble et al., 1997 p17). The contextual inquiry consisted of 300 hours of observations followed by 1300 hours of data analysis over 3 months. Ten different physicians were observed in a variety of clinical settings. From the observations, the researchers generated over 500 requirements, which were then reviewed and prioritised during meetings with the same physicians. This formed the basis of the User Requirements document, which was also 
reviewed with the clinical users to check that requirements had not been misunderstood or misinterpreted before design began.

The authors report that although the $\mathrm{Cl}$ resulted in an extensive list of user requirements for the software, it was challenging to translate these into a workable functional specification and that the repeated consultation with users at this point was crucial to the success of the project.

Doherty et al. (2001) used $\mathrm{Cl}$ to identify user requirements for a new assistive device called 'Cyberlink', a brain-body interface that aims to assist motor-impaired people to communicate by converting eye and facial muscle movements and brain waves into computer inputs. In the case of this device, as well as being the recipient of the device/treatment/care, the patient was also the operator and this meant that a number of different types of requirements had to be satisfied including functionality, comfort and usability. As well as identifying user requirements, the authors of this study report that the $\mathrm{Cl}$ was effective at highlighting potential barriers to the adoption of the new device.

In both of these studies $\mathrm{Cl}$ was used in conjunction with other research methods such as usability tests and focus groups. This reflects of the views of many authors that best practice in user-focused research and development is likely to require using more than a single method or technique (Garmer et al., 2002a, 2002b; ISO 13407; Lin et al., 1998; Salvemini, 1999), this issue will be discussed later on in this paper.

$\mathrm{Cl}$ emphasises the importance of including the real end-user, viewing frontline workers as integral to the design process due to the unique knowledge they have about their own work processes. Historically, the most common application of contextual inquiry has been to the design of computer systems and computer interfaces. However, its successful application to medical technologies as well as the development of office equipment such as fax machines and photocopiers (Rishel et al., 2000) and ATM machines (De Angeli et al., 2004) suggests that it could be used for a wider range of applications including medical devices. $\mathrm{Cl}$ can offer the 
medical device sector valuable, context-specific data obtained from real end-users. It is particularly appropriate at the beginning of the design process or, as $\mathrm{Cl}$ can involve observations and discussions about the overall working environment and wider system, it can be used to look at wider systemic human factors issues as well as equipment and device design. Cl may be particularly useful where current devices are clearly deficient, providing designers with information about the particular ways in which the current devices are failing to meet user requirements.

However, despite the value of $\mathrm{Cl}$ outlined above, there are practical difficulties in carrying it out. Success of this method is dependent upon the observer/interviewer being able to closely shadow and interview workers as they perform their everyday working activities and obtaining this type of access to physicians, nurses, patients and other users of medical devices may be difficult. The relatively intrusive nature of this method may make it unsuitable for use in some healthcare settings, particularly those which involve face-to-face interaction between patient and clinician. In addition, when the worker is involved in complex, demanding or sensitive tasks, it may be dangerous, distracting or inappropriate for the researcher to engage the device operator in the way that is required.

Contextual Inquiry, like all methods that use verbal protocols, relies on the ability of the user to give accurate and comprehensive details of their actions and the reasons behind them; hence developers should be aware of the limits of this type of data. Verbalising tasks as they are being completed may change the way that users perform those tasks, for example if there are a number of ways to perform a task then the user may choose the way that is easiest to explain or which is closest to the 'textbook' method. In addition, the simple addition of talking during a task may change it, for example the speed at which a task is performed. Some processes may be difficult to describe verbally such as tasks that are dependent on perceptual-motor skills. In addition, it can be difficult to elicit all useful information from the user; there are limits to the ability of people to provide a concurrent account of their actions and the reasons for them, $\mathrm{Cl}$ is 
unlikely to access the users' decision making protocols or the tacit knowledge subconsciously used to complete tasks.

\subsection{Cognitive Task Analysis}

Cognitive Task Analysis (CTA), a method for studying the mental processes underlying behaviour, may have a contribution to make to the development of many medical devices. Recent work on the link between increased cognitive load and human error (Horsky et al., 2005; Patel and Kaufman, 1998) has highlighted the need for consideration of the psychological components of healthcare tasks. Militello (1998) compares the healthcare sector with other industries that have adopted CTA and concludes that this technique may be useful in medical device development as "nowhere is an increased cognitive load on humans more visible than in the health care sector" (Militello 1998 p.535).

The aim of a CTA is to identify the cognitive requirements of a task by mapping out the task, identifying and prioritising the critical decision points and investigating the strategies and cues used by users to complete the task successfully. By identifying the cognitive skills and cues required for tasks CTA can inform design, resulting in devices that support the cognitive processes required to perform a task successfully.

Only a small number of examples were identified during the literature review suggesting that CTA is not yet used extensively in medical device development. When discussing the cognitive load placed on users of medical devices an example that is often cited is infusion pumps: machines that are programmed to deliver drugs or nutrients at a certain rate and over a certain time period. Operator error when using infusion pumps has been identified as a major problem that can have serious implications for patient safety (Cohen, 1993; Leape, 1994; Webb et al., 1993). To administer medication to a patient, a nurse will program an infusion pump to automatically deliver the drug over a period of time and this may involve navigating through, and entering data into, a number of different screens. A common problem with this type of task is that the operator often 
has to remember everything they have previously entered which increases the load on the working memory and increases cognitive strain (Obradovich and Woods, 1996).

Obradovich and Woods (1996) and Lin et al. (1998) used CTA to inform the design of new infusion pumps by identifying problems with existing devices. The problems they identified included a lack of feedback provided during programming; a lack of ability for programmers to detect and recover from errors; and confusing and unnecessary complex programming sequences. These problems are likely to increase the cognitive load on the user's working memory and, given that these devices are often used in high pressure working environments such as intensive care units there are clear implications for patient safety. In these studies the results of the CTA were used to inform the design of new devices which were then evaluated by usability tests.

An increasing number of medical devices are now computer-based, therefore, a method such as CTA that examines the cognitive nature of tasks is likely to become increasingly popular, based on the belief that systems and devices are more likely to succeed if the design is intuitive and appropriate for the task at hand.

Traditional task analysis may also be a useful method to use during the design and development of some medical devices. This involves breaking down the task into the individual actions that a user has to perform along with the results (or product) of that action. The task is further analysed to identify factors such as task frequency and difficulty, the importance of each of the actions within the task and the importance of training. This enables designers to identify the critical points in the task and to develop devices or systems to support users at these points. As task analysis is concerned with observing behaviours in terms of actions and results it is more suited to the analysis of physical tasks. This may be of particular use when designing assistive devices for the elderly or disabled.

\subsection{Usability tests}


As previously mentioned, the current focus on human error and patient safety has resulted in an increased awareness of the importance of the usability of medical devices. Garmer et al., (2004 p.86) support this stating that "if the usability of user interfaces was improved, incidents and accidents could be reduced as could the required time to learn how to use new equipment". Usability testing requires users to perform tasks to measure the performance of a device in terms of task time, ease of use or amount of errors. Tests can be performed either in a suitably equipped usability lab or in the field and can either involve a fully functional or non-working prototype.

We found evidence that suggests that usability tests are beginning to be utilised during medical device development both for informing and evaluating design. Cimino et al. (2001) used usability tests to identify problems with a software program for entering data into patient medical records. Outcome measures included the amount and type of error made during the task: for example whether the software misinterpreted or failed to recognise clinical terms and whether the user of the program made an error whilst entering or retrieving data. The results were used to inform the design of a new program with the intention of improving patient safety by reducing the errors associated with entering and retrieving patient data.

Garmer et al. (2004) compared the use of focus groups and usability tests for evaluating ventilator machines. They found that the two techniques elicited different types of information and that neither method detected the full range of requirements. Usability tests were found to be effective at detecting problems that the user was not aware of during interaction with the interface, whilst focus groups were more effective at discovering contextual requirements. This study illustrates the fact that users may not be able to detect and/or communicate all of the problems that may arise in the use of a device for themselves, and also that ideally a number of different methods should be used in any user research to capture different types of data. The users included in this study were nurses with differing amounts of experience and the authors report that a number of useful requirements were identified by novice nurses but were not reported by their more experienced colleagues. Identifying these types of requirements meant 
that the new device was designed to take into account the needs of those learning to use the system: an important component of usability. This study emphasises the importance of ensuring an inclusive sample of users are investigated so that the limitations of failing to incorporate the experiences of small but significant sub-sections of the user population are avoided.

As well as informing design, usability tests have also been used to evaluate new medical devices to demonstrate improvements over existing models. As discussed above Lin et al. (1998) used usability tests to evaluate a new design of infusion pump. Outcome measures included the time to complete the tests, the number of errors made and subjective mental workload ratings.

Although expensive and time consuming, the optimum method of applying usability tests is to perform a number of tests throughout an iterative design procedure (ISO 13407 1999). Two papers by Garmer et al. (2002a, 2002b) describe a long-term study of infusion pumps which included a number of iterations of usability tests as part of a general user centred design approach. The authors of these papers were sensitive to the effect that the experience of the users, in this case nurses, would have on their performance in the usability tests and included nurses with a wide-range of experience. They found that the more experienced nurses were much more critical of the prototype and were more likely to offer suggestions for improvements. However, novice users also provided useful data as they were more likely to make errors. The authors make the point that when making errors both novice and expert users can often blame themselves for insufficient knowledge or competence rather than seeing deficiencies in the product. Developers should be sensitive to this and encourage and motivate users to be critical of the device rather than themselves. Indeed it should be kept in mind that all methods that rely on users' perceptions are subject to bias in terms of culpability for errors, either in tendencies to blame the system/device or blame themselves. Garmer et al. conclude that a multi-faceted approach is essential to fully understand user capabilities and tasks in the context 
of the work environment. Suitable usability goals can then be set to allow empirical testing and, if necessary, further refinement of the device.

\subsection{Heuristics}

Heuristic evaluation involves a small group of evaluators testing a prototype device against design principles (heuristics) in order to identify usability problems. Principally used for evaluating computer user interfaces, heuristic evaluation should be performed as part of an iterative design and evaluation process.

Zhang et al. (2003) describe their experience of using heuristic evaluation during the design and development of an infusion pump and discuss whether this method, developed for humancomputer interfaces, can be applied more broadly to medical device development. Based on previous work and their own experience, the authors compiled fourteen usability heuristics that user-interfaces should adhere to. These included 'users should be informed about what is going on with the system through appropriate feedback and display of information'. The evaluators, who were students of human-computer interaction, then performed a walk-through of the interface by performing a number of tasks with the device with the usability heuristics in mind. Each evaluator recorded which heuristics had been violated, and if so, how severe the violation was.

The authors recognise that this technique will not pick up all usability problems, they estimate that any one evaluator may pick up approximately $35 \%$ of usability issues and that 5 evaluators will pick up approximately $60-75 \%$. These estimates, however, are based on work on computer user interfaces and further research is necessary to determine the extent to which they can be extrapolated to general medical devices. Zhang et al. conclude that heuristic evaluation is an appropriate discount usability technique that could be applied to medical devices as it can detect key usability issues in a short period of time for a limited cost. However, this was based on an infusion pump which is a computer-based medical device and the authors recognise that many of the usability issues were generic $\mathrm{HCl}$ issues rather than issues relating to its medical application. The appropriateness of this technique for non-computer based medical devices is 
not known and it is questionable whether it would be appropriate to use non-medical personnel to evaluate medical devices. However, this technique could have a place in medical device development as an initial usability test that could be performed in-house early on in the development stage to identify and resolve initial problems before performing an extensive usability test on a later prototype. This method may also be useful when there are confidentiality issues that may be breached by testing a product on true end-users or contextual evaluation is impossible. However the evaluation is only as good as the heuristics against which the device is assessed and these should be validated for completeness, validity and potential bias.

\subsection{Cognitive walkthrough}

A cognitive walkthrough $(\mathrm{CW})$ is similar to a heuristic evaluation in that it is performed by expert evaluators rather than actual end-users, though it is a less structured method with no predetermined, formal rules or heuristics to judge the device against. In a cognitive walkthrough, the focus is on learning through exploration rather than being restricted only to pre-defined factors. In practical terms, however, the way in which heuristic evaluation and cognitive walkthrough are carried out may be similar. The evaluator defines the sequence of actions required to perform a certain task and will then step through that sequence to check for potential usability problems with the main focus often how easy a system would be to learn for a novice user. Although this method may not use an explicit list of heuristics the evaluator will inevitably use their knowledge on usability issues which could be seen as 'instinctive heuristics'. As with heuristic evaluation, using a CW may be a useful method to employ when there are confidentiality issues or difficulties in accessing users.

We identified two papers that report the use of $\mathrm{CW}$ for computer-based medical applications: a medical software program (Patel and Kaufman 1998) and a system for patients to monitor their health from within their homes (Kaufman et al., 2003). Kaufman et al. performed a cognitive walkthrough to determine what cognitive demand the computer system would place on a predominately elderly target population, stating that "elderly users of a system are likely to have 
a lower tolerance for excessive memory or attentional demands" (Kaufman et al., 2003 p.47). As a result of the $\mathrm{CW}$, a number of changes were made to reduce the strain on the users and this study illustrates the need to be sensitive to the special requirements of the target users of a device. The authors, however, do acknowledge the difficulty of identifying all problems that may be encountered by users and for this reason supplemented the CW with usability tests with real end-users. These additional tests identified a number of additional problems that were not anticipated by the developers during the $\mathrm{CW}$.

Cognitive walkthrough is a low investment technique ideally suited to the early identification of design problems. It is quick and low-cost and therefore a number of iterations can be performed early in the design. An advantage is that it requires the designer to think like a user but with the benefit of usability knowledge. In some cases, the designer will be more sensitive to design problems than a true user: as observed already, users, especially novice users, can tend to ascribe difficulties and errors to their lack of knowledge rather than the shortcomings of a product. However, developer bias and lack of ability to put themselves in the users' shoes is a traditional shortcoming of designer/developer assessment techniques however and should never be solely relied upon.

The main disadvantage of $\mathrm{CW}$ is that it is very difficult for a designer to truly think and act like a user, especially a novice user. The literature recognises this with most papers recommending the need for further, extensive usability testing with true end-users. The cognitive walkthrough is suited for the identification of large usability problems early on in the evaluation process but should be complemented with usability tests.

\subsection{Focus Groups}

Focus groups are a very popular method of data collection in many industries due to the relatively low cost and time commitment required and are frequently used within a user-centred design approach (Anderson, 2002; Doherty et al., 2001, Garmer et al., 2004; Gould and Lewis, 1985; Mello, 2001). Focus groups consist of a facilitated discussion of a topic and aim to 
discover the opinions of the group of individuals; they do not capture the individual opinions of each participant. Focus groups can be used throughout device development, for both formulating and evaluating designs, and therefore developers may be attracted by the thought that they may only have to learn how to perform one research method making this method even more appealing.

We identified a number of examples of the use of focus groups in medical device development. Batavia and Hammer (1990) used a focus group approach for the design of new assistive devices. Long-term users of assistive devices took part and the focus groups resulted in a list of possible features for inclusion in new devices. A panel of different long-term assistive device users then prioritised these features by the use of a Delphi technique (see section 3.7). The authors report that this approach was successful in obtaining user requirements and resulted in a number of important recommendations for device design. This approach of identifying requirements in focus groups and then refining and prioritising them using a technique such as Delphi was also used by Lane et al. (1997) for the re-design of a charger for wheelchair batteries. The authors report that this method was effective at identifying and obtaining consensus on the requirements of the device users and identified a number of issues not normally considered during product evaluation.

Barrett and Kirk (2000) considered the use of focus groups with elderly and/or disabled participants and whether any particular strategies should be employed when engaging with these types of users. The authors argue that there may be some particular difficulties in running focus groups with these types of users: the general challenges of formulating appropriate discussion questions and the role of the moderator in facilitating discussion may be complicated when dealing with such participants. A number of strategies are recommended which, although particular relevant for elderly or disabled participants, may be useful for all focus groups. These include providing background information beforehand, keeping questions simple and using language that participants will understand. In addition, the moderator must be able to communicate clearly, listen carefully and sensitively guide the discussion, for example, ensuring that only one participant speaks at a time and preventing interruptions. 


\subsection{Delphi Technique}

As well as identifying user requirements the development process must also enable these to be prioritised. The large number of different users and stakeholders in medical device development can make this especially complex. The Delphi technique may be an appropriate method to use for prioritising user requirements. This method aims to obtain a consensus on an issue by asking 'experts' to provide opinions or ratings. The results of this are used to formulate another round of questions during which the experts are made aware of how their responses compared with those of the other respondents. In the case of medical devices, experts may include recipients (patients), operators (carers, patients, staff), procurement, maintenance, trainers or all of these groups, and the success of this method will be dependent upon the correct choice of stakeholders.

The Delphi technique normally consists of a number of rounds of questions to progressively identify, clarify and expand on issues and ideas. The first round usually consist of general questions to gain a broad understanding of the views, opinions or needs of the experts. The responses from this round are collated and summarised and this forms the basis of the next round of questions which will investigate further or clarify the issues raised in round 1. Again the results are collated and used to formulate a third (and usually final) set of questions which will generally consist of obtaining a consensus on the relative importance of the factors identified in the previous rounds. A key aspect of Delphi is the anonymised feedback that the experts receive between each round of questioning; general feedback is provided to the experts between rounds and individuals are given the opportunity to revise judgements as a result of the feedback.

An example of the use of the Delphi technique in medical device development is provided by Batavia and Hammer (1990) who, as described above, used the technique in conjunction with focus groups to identify needs and requirements for new assistive devices. Focus groups were performed with a large number of users of existing assistive devices to identify areas where 
these were deficient which resulted in a list of possible features for inclusion in new devices. A panel of experts, which consisted of a sample of long-term assistive device users, then performed a number of rounds of questioning to prioritise the requirements. The authors report that this approach was successful in obtaining user requirements and resulted in a number of important recommendations for device design such as the high importance to users of a long battery life for powered wheelchairs.

The Delphi technique may be a useful option when wanting to obtain a consensus of opinion on a complex issue. It may also be useful when a face to face meeting may not be appropriate, for example in situations where publicly voicing a contrary or critical opinion may be unlikely due to a particular group dynamic or the type of users involved. The lack of face to face meeting means that Delphi may be useful when it is difficult to get many users together at the same time and with the increasing popularity of email this method is becoming quicker, cheaper and easier to perform

\subsection{Summary}

There are a large number of methods available for developers wishing to capture user requirements during medical device development. The decisions on which to use will depend on a number of factors such as the point in development the device is at; the type of users involved; the expertise of the research; the type of information required and, most importantly, the time and money available (table 3). As developers are under pressure to minimise cost and time to the launch of their product, it is unlikely to be practical to carry out rigorous user research using traditional scientific methods. It is here that the ergonomics research methods discussed in this paper are particularly appropriate as they have been developed in response to the practical constraints of product development.

\section{Factors affecting User Requirements Research}


We identified a number of themes that will affect the choice of method for a particular application. Many of these factors will be common to all product development such as the point in the product lifecycle the device is at, the type of users that are to be studied and the cost, time and expertise available for the user research. Many principles that apply to general product development also apply to medical device development such as the use of open-ended scoping methods during the concept stage and more focused and evaluative methods during design and development. However, the nature of the healthcare sector means there are additional factors to be considered when planning user requirements research in this field.

As there may be multiple user groups for any one device developers must be aware of all of these, know their characteristics and abilities and where and how they will use the device. This will vary according to a number of factors including whether the device will be used in a hospital, ambulance, clinic or in the patient's home; who the primary operator of the device is, e.g. doctor or nurse and who is paying for the device: a healthcare trust, the patient or an insurance company.

If identifying the multiple users of a potential device is complex, then accessing them can be particularly difficult in the healthcare domain. The regulatory requirements surrounding medical device development are frequently a barrier to studying and including users. Certain regulations must be met before devices can be tested by real users and, once a device has met regulations, developers can be reluctant to make changes that would require re-submission, given the time and financial cost of putting devices through regulatory assessment. In addition to this, there are usually strict research governance procedures that have to be completed before patients or health care professionals can be contacted. For example, the process of applying for ethical permission for research in the UK NHS (via The National Research Ethics Service, previously the Central Office of Research Ethics Committees) is a particularly lengthy and complicated procedure. All research involving NHS staff or patients requires ethical review, whether interventional or not, and this has previously been identified as a barrier to research (Wald 2004). Studies involving children or vulnerable adults or their data require the researcher 
to have had a standard Criminals Records Bureau (CRB) disclosure completed within the last six months prior to the start of the project. For medical device development issues such as these can often mean that usability or identifying 'softer' user requirements are seen as a lesser priority compared with issues such as proving clinical efficacy.

There are a number of additional factors that make user requirements study challenging:

- Context and sampling. Medical devices are used by a number of very different users, within different environments, for the treatment of different conditions and with different levels of prior, relevant experience, and in conjunction with other devices and equipment. An appreciation of the likely impact of these factors during development is essential. Wider organisational issues that influence the effectiveness, usability and safety of a device such as working procedures, team work, communication, safety culture and management attitudes must also be considered. An inability to access a sample of users that is representative of all of these contextual factors will lead to incomplete or biased data.

- Field research may be entirely inappropriate. Due to the difficulty of obtaining access to the real clinical environment, for example because of research governance, confidentiality or privacy issues, alternatives to field research may have to be considered. Medical simulation centres, which are becoming increasingly popular for training medical staff (Blackburn \& Sadler 2003), may also be used to provide realistic clinical scenarios in which devices can be evaluated. However, no matter how realistic a scenario they may provide, simulation centres should not entirely replace research in the real environment where the compatibility of the device with other equipment as well as environmental effects can be studied.

\section{- It may prove more difficult to obtain users during certain phases of product} development. Developers may have to consider using proxies in the place of real users particularly early on in the design process. For example, using healthy 
participants to test a device for usability instead of patients, or asking clinicians to provide the views of their patients, e.g. 'what factors do your patients say are important to them?' In these circumstances methods such as walkthroughs or heuristic evaluations which can be performed in house, using developers or other staff as proxies, may be useful to identify and fix large problems early in development. However, this type of evaluation, although a traditional route to addressing user issues, is fraught with bias and should never entirely replace testing with representatives of the target users. The use of proxies may also be useful in circumstances when the target users of a potential device may have difficulty expressing their own opinions: for example very young children or those with severe mental impairments, although it is critically important that in these cases the appropriate proxy is chosen to represent the user. Performing early evaluations of products in-house also ensures that confidential details of new technologies are not compromised.

- It is recommended that more than one research method is used at each stage of product development. As different methods are effective at identifying different types of data (Garmer et al 2004), it is recommended that more than one method is used during product development in order to capture a range of data (ISO 13407 1999, Lin et al 1998, Salvemini 1999, Garmer et al 2002a, 2002b).

In addition to access to users, the intrusiveness of some methods discussed in this paper may mean that they are unsuitable for use within a medical setting or may have to be modified: for example when using contextual inquiry it may be inappropriate for the researcher to ask questions of the users whilst they are performing tasks or during patient consultations or certain surgical procedures. In such cases questions and discussion may need to be conducted after the task has finished.

To determine how accessible ergonomics methods are to those working in device development we compared the findings from our literature review with other reviews of ergonomics methods. In 1998 Stanton and Young conducted a survey into the methods used by ergonomics in the field of 
human computer interaction. They found that although there are over 60 different ergonomics methods available to product developers, most practitioners use just a small number of well established methods and concluded that "most methods are used by their inventors only" (Stanton and Young, 1998 p41). Our literature review supports this finding as we identified more than thirty methods that have been used to capture user requirements, however, many of these were just slight variations of other more established methods. The majority of papers identified utilised the methods described in this paper: these being commonly used, well established techniques.

The picture is further confused by the fact that so many different terms are used by practitioners to describe the research methods they use, for example, what one person calls contextual inquiry another may call shortened or focused ethnography. This makes it difficult for product developers, who may wish to use ergonomics methods during design and development but may be uncertain as to which to choose for their specific application: the slight variations between the methods and the different names that they are given will make those decisions more difficult and may prove a barrier to adopting ergonomics methods.

\section{The Role of Ergonomics}

Should a role of ergonomics be to make its methods accessible to product developers and designers to ensure ergonomics principles are applied as widely as possible, or should we be concerned over the rigour with which these methods could be applied by those with limited human factors training? This is a topic which has been identified as a key challenge for ergonomics, as Wilson states, "consistency on the balance between professionalism and protection on the one hand, and growth and spread on the other, will be difficult to achieve, but vital" (Wilson, 2000). 
Like many manufactured products, a huge proportion of medical devices are developed by small companies who are unlikely to have the resources to employ ergonomics consultancies or staff with specific ergonomics expertise. This should not be a barrier to adopting ergonomics principles. The role of the ergonomics community should be to provide resources and knowledge that allow product developers, engineers, academics and clinicians to make decisions on the best use of their resources for studying user requirements during the development of their product. Even if these resources are extremely limited it is preferable that a small, well-planned and well conducted study is performed rather than no attempt to capture user requirements, which may be the realistic alternative. Developers are more likely to adopt ergonomics principles if they believe that doing so will benefit them in terms of either improved sales, a better quality product or a more efficient development pathway: "While pressure can be put on industry to encourage it to focus on user-centred design practice, industry is unlikely to respond to abstract directives or inducements. What is needed, therefore, is a body of exemplar case studies and demonstration projects that show how such an approach can lead to better and more competitive products" (Buckle et al, 2006).

The MATCH project is currently working on a number of case study projects, in collaboration with a number of partners from within industry, healthcare and academia, to investigate the development of real medical devices. As well as studying how user requirements are currently collected and incorporated into medical device design, we are also working alongside device developers to implement rigorous, yet realistic, user research during product development. The value, impact and perceived value of the user research - to both the developers and users - will be studied throughout product development.

\section{Conclusion}

Apart from the recent focus on usability of devices, in particular of infusion pumps, little published work exists on the ergonomics aspects of medical device development. In particular there is little advice available to developers on which issues to consider during design and 
development or recommendations for good practice in terms of the methods and approaches needed to capture the full range of user requirements. Industry needs guidance to help apply the most appropriate methods at the most appropriate stages of the product cycle, and with the most appropriate groups of users.

However, the labour intensive nature of many of the methods used to capture user requirements means without cogent evidence of the cost-benefit of ergonomics or enforced safety that it is only likely to be used where there is a strong commitment to maximising usability. This raises issues common to medical device development of who they are designing for: the patient, the worker (clinician) or the purchaser. Undoubtedly it would be extremely useful for designers of any device to observe real-world use of the products, however this would depend on both industry and the healthcare sector recognising that clinical need should be the push for new technology.

Although the literature review provides an indication of the methods that have been used to capture user requirements it is not possible to determine from this what the current practices within the medical device industry actually are. Published papers do not necessarily reflect methods in practice: some methods may be so well established that there is no need for further research into their use; methods discussed in the scientific literature may rarely be applied in practice; and current industrial practices may be commercially confidential. Therefore close consultation and collaboration with industry is vital if any new research is to reflect and improve on current practices. Our aim should be to make our scientific knowledge available to strengthen the innovative capacity of those working in medical device development.

This review of published work is not a review of the methods that could be applied to medical devices and there are of course numerous other methods that could be applied that are not discussed here. The next step is to look widely at ergonomics methods and approaches and to apply these in case study projects with the ultimate aim of producing an industry-focused guide that will support and encourage the application of ergonomics principles in medical devices. 


\section{Acknowledgements}

The authors acknowledge support of this work through the MATCH Programme (EPSRC Grant GR/S29874/01), although the views expressed are entirely their own.

We would also like to acknowledge and thank our MATCH colleagues: Mala Bridgelal Ram, Natasha Campling (nee Browne), Dr Patricia Grocott and Heather Weir from Kings College London; and Professor lan Robinson and Dr Sarwar Shah from Brunel University.

\section{References}

AAMI HE74: 2001. Human Factors Design Process for Medical Devices.

Anderson, G.J., 2002. Shaping the Future: Using Voice of the Customer Methodology to Develop Inhaler Design. Resp. Drug Del. 8, 1-10.

Barrett, J. and Kirk, S., 2000. Running focus groups with elderly and disabled elderly participants. Appl. Ergon. 31, 621-629.

Batavia, A.I. and Hammer, G.S., 1990. Toward the development of consumer-based criteria for the evaluation of assistive devices. J. Rehab. Res. Dev. 27, 425-436.

Blackburn, T. and Sadler, C., 2003. The role of human patient simulators in health care training. Hosp Med. 64, 677-681.

BS 7000-6:2005 Design management systems. Managing inclusive design. 
Buckle, P., Clarkson, P.J., Coleman, R., Lane, R., Stubbs, D., Ward, J., Jarrett, J. and Bound J., 2003. Design for Patient Safety: a System-wide Design-led Approach to Tackling Patient Safety in the NHS. Department of Health Publications, London. ISBN 1-84182-7657.

Buckle, P., Clarkson, P.J., Coleman, R., Ward, J. and Anderson, J. 2006. Patient safety, systems design and ergonomics. Appl. Ergon 37, 491-500.

Cimino, J.J., Patel, V.L. and Kushniruk, A.W., 2001. Studying the human-computer-terminology interface. J. Am. Med. Inform. Assn. 8, 163-173.

Coble, J.M., Karat, J. and Kahn, M.G., 1997. Maintaining a focus on user requirements throughout the development of clinical workstation software. Proceedings of the 1997 Conference on Human Factors in Computing Systems, CHI, Mar 22-27 1997. ACM, New York, NY, USA, Atlanta, GA, USA, 170.

Cohen, M.R., 1993. Preventing errors associated with P.C.A. pumps. Nursing. 23, 17.

De Angeli, A., Athavankar, U., Joshi, A., Coventry, L. and Johnson, G.I., 2004. Introducing ATMs in India: A contextual inquiry. Interact. Comput. 16, 29-44.

Doherty, E., Cockton, G., Bloor, C. and Benigno, D., 2001. Improving the performance of the Cyberlink mental interface with the "Yes/No program". Conference on Human Factors in Computing Systems CHI 2001 Anyone. Anywhere, Mar 31-Apr 5 2001. Seattle, WA, 69.

Garmer, K., Liljegren, E., Osvalder, A. L. and Dahlman, S., 2002a. Arguing for the need of triangulation and iteration when designing medical equipment J. Clin. Mon. Comp. 17, 105-114

Garmer, K., Liljegren, E., Osvalder, A. and Dahlman, S.. 2002b. Application of usability testing to the development of medical equipment. Usability testing of a frequently used infusion pump 
and a new user interface for an infusion pump developed with a Human Factors approach. International J. Ind. Ergon. 29, 145-159.

Garmer, K., Ylven, J. and Karlsson, I.C.M., 2004. User participation in requirements elicitation comparing focus group interviews and usability tests for eliciting usability requirements for medical equipment: A case study. Int. J. Ind. Ergon. 33, 85-98.

Gould, J.D. and Lewis, C., 1985. Designing for Usability: Key principles and what designers think. Communications of the ACM. 28, 300-311.

Holtzblatt, K. and Beyer, H., 1993. Making customer-centered design work for teams. Commun. ACM. 36, 93-99.

Horsky, J., Zhang, J. and Patel, V.L., 2005. To err is not entirely human: Complex technology and user cognition. J. Biomed. Inform. 38, 264-66.

IEC 60601-1:2004. General Requirements for Safety of Electrical Medical Equipment.

IEC 60601-1-6: 2004. General Requirements for Safety of Electrical Medical Equipment Collateral Standard: Usability. IEC 60601-1-8: 2004. Collateral Standard: General requirements, tests and guidance for alarm systems in medical electrical equipment and medical electrical systems.

ISO 13407: 1999. Human-centred design processes for interactive systems.

ISO 14971: 2000. Medical Devices - Application of Risk Management to Medical Devices. 
Kaufman, D.R., Patel, V.L., Hilliman, C., Morin, P.C., Pevzner, J., Weinstock, R.S., Goland, R., Shea, S. and Starren, J., 2003. Usability in the real world: assessing medical information technologies in patients' homes. J. Biomed. Inform. 36, 45-60.

Lane, J.P., Usiak, D.J., Stone, V.I. and Scherer, M.J., 1997. Voice of the customer: Consumers define the ideal battery charger. Assis. Technol. 9, 130-139.

Leape, L.L., 1994. Error in medicine. JAMA: J. Am. Med. Assn. 272, 1851-1857.

Liljegren, E., Osvalder, A. and Dahlman, S., 2000. Setting the requirements for a user-friendly infusion pump. Proceedings of the XIVth Triennial Congress of the International Ergonomics Association and 44th Annual Meeting of the Human Factors and Ergonomics Association, 'Ergonomics for the New Millennnium', Jul 29-Aug 4 2000. Human Factors and Ergonomics Society, San Diego, CA, United States, 132.

Lin, L., Isla, R., Doniz, K., Harkness, H., Vicente, K. J. and Doyle, D.J., 1998. Applying human factors to the design of medical equipment: Patient-controlled analgesia. J. Clin. Mon Comp. 14, 253-263.

Mello, S., 2001. Right process, right product. Res. Tech. Manage. 44, 52-58.

Militello, L.G., 1998 Learning to think like a user: using cognitive task analysis to meet today's health care design challenges. Biomed. Instrum. Techn. 32, 535-540.

Obradovich, J.H. and Woods, D.D., 1996. Users as designers: how people cope with poor $\mathrm{HCl}$ design in computer-based medical devices. Hum. Factors. 38, 574-592.

Patel, V.L. and Kaufman, D.R., 1998. Medical informatics and the science of cognition. J. Am. Med. Inform. Assn. 5, 493-502. 
Rishel, K.C., Grindstaff, R. and Beckman, O., 2000. Understanding the needs of international customers: Applying the contextual inquiry method in Asia. Proceedings of the XIVth Triennial Congress of the International Ergonomics Association and 44th Annual Meeting of the Human Factors and Ergonomics Association, 'Ergonomics for the New Millennnium', Jul 29-Aug 42000. Human Factors and Ergonomics Society, San Diego, CA, United States, 662.

Salvemini, A.V., 1999. Challenges for user-interface designers of telemedicine systems. Telemed. J. 5, 163-168.

Sawyer D., 1996. Do it by design: an introduction to human factors in medical devices. US Department of Health and Human Services Food and Drug Administration (Center for Devices and Radiological Health)

Shefelbine, S., Clarkson, P.J. and Farmer, R., 2002. Good design practice for medical devices and equipment - requirements capture, vol. 1. University of Cambridge Engineering Design Centre/University of Cambridge Institute of Manufacturing.

Stanton, N.A. and Young, M.S., 1998, Is utility in the mind of the beholder? A study of ergonomics methods. Applied Ergonomics. 29, 41-54.

Wald, D.S., 2004. Bureaucracy of ethics applications. BMJ. 329, 282-284.

Webb, R.K., Russell, W.J., Klepper, I. and Runciman, W.B., 1993. The Australian Incident Monitoring Study. Equipment failure: an analysis of 2000 incident reports. Anaesthesia and Intensive Care. 21(5), 673-677.

Wilson, J.R., 2000. Fundamentals of ergonomics in theory and practice. Appl. Ergon. 31, 557567. 
Zhang, J., Johnson, T.R., Patel, V.L., Paige, D.L. and Kubose, T., 2003. Using usability heuristics to evaluate patient safety of medical devices. J. Biomed. Inform. 36, 23-30. 